\title{
Health System Preparedness in Tackling the COVID- 19 in Nepal: a Qualitative Study Among Frontline Healthcare Workers and Policymakers
}

Nipun Shrestha

Victoria University

Shiva Raj Mishra

Nepal Development Society

Saruna Ghimire

Miami University, Oxford, USA

Bishal Gyawali

University of Copenhagen

Sujan Babu Marahatta

Manmohan Memorial Institute of Health Sciences

Smriti Maskey

Fort Worth, Texas

\section{Sushila Baral}

Manmohan Memorial Institute of Health Sciences

Nilima Shrestha

Manmohan Memorial Institute of Health Sciences

\section{Rakesh Yadav}

Nepal Community Health and Development Center

Bipin Adhikari ( $\sim$ biopion@gmail.com )

Nepal Community Health and Development Centre https://orcid.org/0000-0001-8981-3910

\section{Research article}

Keywords: COVID-19, health system, health system preparedness, health workers, Nepal

Posted Date: August 12th, 2020

DOI: https://doi.org/10.21203/rs.3.rs-49448/v1

License: (c) (1) This work is licensed under a Creative Commons Attribution 4.0 International License.

Read Full License 
Version of Record: A version of this preprint was published at Disaster Medicine and Public Health Preparedness on June 18th, 2021. See the published version at https://doi.org/10.1017/dmp.2021.204. 


\section{Abstract}

\section{Background}

Rapidly growing coronavirus disease 2019 (COVID-19) pandemic has brought unprecedented challenges to the health system in Nepal. The main objective of this study was to explore the characteristics and drivers of the health system preparedness towards COVID-19 and its impacts on frontline health care workers (HCWs) in Nepal.

\section{Methods}

Semi-structured interviews (SSIs) were conducted among $32 \mathrm{HCWs}$ who were involved in clinical care of COVID-19 patients and four policymakers who were responsible for COVID-19 control and management at central and provincial level. Participants were included from all seven provinces of Nepal. All SSIs were conducted through telephone or internet based tools such as Zoom and Skype. All interviews were audiorecorded, transcribed into English, and coded using inductive and deductive approaches.

\section{Results}

Both HCWs and policymakers reported failure to initiate pre-emptive control measures at the early stages of the outbreak as the pivot in pandemic control. Although several measures were rolled out when cases started to appear, the overall health system preparedness was low. Specifically, there was inadequate personal protective equipment for HCWs, insufficient isolation beds for patients, poor coordination between the three tiers of governance, and poor engagement of the private sector. Additionally, HCWs experienced various degrees of stigma because of their profession and yet were able to maintain their motivation to continue working in the forefront.

\section{Conclusion}

This study identified poor preparedness of the health system in the course of Nepal's COVID-19 response embedded in the governance. Specifically, the lack of human resources, inadequate logistic chain management and laboratory facilities for testing COVID-19 appeared to have jeopardized the health system preparedness and escalated the pandemic in Nepal. Despite the poor preparedness, and health and safety concerns, HCWs maintained their motivation. Urgent efforts are required to reform the governance and coordination mechanism in addition to incentivizing the HCWs for the current and future pandemics.

\section{Background}

In December of 2019, a clustered outbreak of pneumonia, later named as coronavirus disease 2019 (COVID-19), was reported in Wuhan, China [1]. As of June 15, over 8 million confirmed cases and 436 thousand deaths related to COVID-19 had been reported globally [2]. The first case of COVID-19 in Nepal was reported on January 13, 2020 [3]. While the morbidity and mortality attributed to COVID-19 continued 
to escalate in other countries, no new cases were reported in Nepal until the second week of March 2020. Currently, COVID-19 cases are rapidly increasing in Nepal, and as of June 17, 2020, there were 7, 177 confirmed cases reported from all seven provinces of Nepal and 20 deaths (Figure 1) [2].

Nepal's health system fundamentally suffers from constrained resources; and universal health coverage is yet to be attained, particularly in the rural regions [4-6]. Nepal's fragile health system is struggling to manage the rising pandemic due to COVID-19; specifically in screening a large number of community cases, establishing diagnosis, and accommodating treatment at the available (limited) health facilities [7]. As a part of the public health measure, Nepal exercised a series of complete lockdowns since March $24,2020[8]$.

The country has made impressive progress in expanding the number of testing centers from one to 18 in the past few months [9]. Yet, the existing diagnostics facilities for testing and confirming COVID-19 cases are inadequate, and the provision of such services at the peripheral parts of the country is still far-fetched [7]. Twenty-five hospitals in Nepal are designated as the hub hospitals that provide a dedicated space with isolation facilities for COVID-19 patients but most hospitals in Nepal are mostly unprepared for the surge of COVID-19 infections mainly due to lack of resources that includes limited intensive care units (ICUs), isolation wards, and human resources $[5,7,10]$.

While health system in Nepal is constrained by the number of HCWs, their functionality is further restricted and discouraged by poor preparation, specifically due to lack of adequate personal protective equipment (PPE) [11]. The constraints occurring at health service and personal level can have a huge psychological impact on HCWs resulting into stress, anxiety and depression [12]. The recent global reports of high infection and deaths among HCWs in addition to stress and pressures at the workplace are alarming and can have devastating consequences to the denominator population. The main objective of this study was to explore the characteristics and factors affecting Nepal's health system preparedness towards COVID-19 and its impact on HCWs.

\section{Methods}

\section{Design}

This was a qualitative study with Nepali frontline HCWs and policymakers using a standard consolidated criteria for reporting qualitative studies (COREQ) guideline (Appendix 1) [13]. Given the limited research on the topic in Nepal, a phenomenological approach was used to explore the topic in-depth, from the perspectives of frontline HCWs and policymakers who were directly involved in the management of the current pandemic.

Institutional Review Board at Manmohan Memorial Institute of Health Sciences, Kathmandu, Nepal (Registration number: 76/133) approved the study. Interviewers briefed about the study, their profile including reasons for the study (study objectives and procedures, and of potential benefits and harms in participation) with individual respondents before fixing the appointments. Participants were informed of 
voluntary participation and their rights to withdraw from the interview at any point or to refuse to answer any questions. Participants who provided verbal consent and permission to audio-record the interview were included in the study. Data were de-identified, and any identifying contents were replaced with specific codes throughout the transcription process to ensure participants' anonymity and confidentiality. Transcriptions were shared only among researchers in this study.

\section{Study participants}

Participants from all seven provinces of Nepal, who were directly involved in the clinical (e.g., HCWs) or non-clinical (e.g., policymakers) management of COVID-19 outbreak, were included in this study (Table 1). Our sampling approach was based on the basic tenet of qualitative research, where respondents are selected based on their involvement and experience about the phenomena of interest (purposive selection) [14]. We interviewed $32 \mathrm{HCWs}$ (17 medical doctors and 15 nurses) from tertiary healthcare institutions and four policymakers. Among all approached candidates, appointments were made based on their free time. Repeated follow-ups and adjustment to appointments were made to interview the candidates, however, no repeat interviews were carried out. None of the candidates refused to participate. The policymakers were from the Ministry of Health and Population at the federal level and the Ministry of Social Development at the provincial level and were involved in devising or implementing COVID-19 management policies at the respective level. Based on the principles of 'data saturation', sample size for this study was deemed sufficient when no new data/themes emerged from further interviews [15].

\section{Table 1: Socio-demographics of participants}




\begin{tabular}{|c|c|c|}
\hline Characteristics & Categories & Frequency (\%) \\
\hline Age range & Continuous & $24-58$ years \\
\hline \multicolumn{3}{|l|}{ Gender } \\
\hline & Male & $19(50 \%)$ \\
\hline & Female & $18(50 \%)$ \\
\hline \multicolumn{3}{|l|}{ Provinces } \\
\hline & Province-1 & $3(8 \%)$ \\
\hline & Province-2 & $4(11 \%)$ \\
\hline & Bagmati Province & $22(61 \%)$ \\
\hline & Gandaki Province & $3(8 \%)$ \\
\hline & Province-5 & $1(3 \%)$ \\
\hline & Karnali Province & $2(6 \%)$ \\
\hline & Sudurpaschim Province & $1(3 \%)$ \\
\hline \multicolumn{3}{|l|}{ Occupation } \\
\hline & Doctors & $17(47 \%)$ \\
\hline & Nurses & $15(42 \%)$ \\
\hline & Policymakers & $4(11 \%)$ \\
\hline
\end{tabular}

\section{Data collection and interview guide}

Semi-structured interviews were conducted, between March 2020 and May 2020, remotely using telephone or internet-based tools that included Skype, Zoom, and Facebook messenger. All interviews were audio-recorded. All respondents participated alone in the interviews when they were off the duty, either at their home or at a peaceful location near their office. Such a remote interview method also enabled us to approach and include a diverse range of participants from different geographic locations within Nepal. A total of 36 respondents participated in this study (Table 1). Two female research assistants: SB, and NilS conducted the interviews who are pursuing Master's degree in Public Health, and were trained in qualitative research by NS, SBM and BA who have MD, PhD and MD, DPhil respectively with extensive experience and expertise in qualitative research.

An interview guide, consisting of a list of relevant themes and corresponding open-ended questions, was developed based on the review of literature and input from the experts. The interview guide was developed for this study and has not been published elsewhere (Appendix 2). The interview guide was pilot tested among two health workers by NS and BA, and were amended for clarity and thematic 
revisions. The guide helped the interviewer to conduct the interview based on the research topic/themes. After a brief video-interactions with the respondents, the interviews were switched to audio for clarity and were conducted in the local language (Nepali) which lasted between 45 to 60 minutes. The interviewer also noted down the major and prominent themes during the interview, which aided the data analysis.

\section{Data analysis}

All interviews were transcribed and translated verbatim into English. Transcripts were assessed for accuracy by cross-checking with the audio-recordings. All data were coded line by line in qualitative data analysis software NVivo 12 (QSR International, Doncaster, Australia). The initial codebook was deductively developed based on the interview guide and was revised to include the emerging codes using the inductive approach. Two investigators (NS and BA) independently checked the transcribed data for accuracy and coded the data. Consensus regarding the nature and coding of final themes was reached through regular team meetings during the data analysis. Regular debriefings were conducted within the study team to discuss the final themes and their interpretations by NS and BA. Findings relevant to the research question are supported by direct quotes of the participants and are presented below.

\section{Results}

\section{Overview of findings}

Although the initial outbreak of COVID-19 in China and elsewhere, as well as its own first confirmed case back in December, provided Nepal adequate time and opportunity for preparedness, the government of Nepal and health policymakers did not seem to anticipate the scale of the pandemic in terms of resources and management it required (Figure 2). Few preparedness measures were taken that included initiation of Incidence Command Activating System for COVID-19 as a health emergency; implementation of complete lockdown; designation and establishment of COVID-19 dedicated healthcare facilities; and preparing curative and preventive guidelines for the management of COVID-19 cases. Nonetheless, the preparedness was deemed futile due to significant gaps that included: 1. Lack of integrated approaches and coordination (between a. federal, provincial, and local level; and b. public and private healthcare facilities) for management of COVID-19; 2. Inadequate number of frontline HCWs; and 3. Constraints in equipment/resources such as space, PPE, and facilities. Despite these constraints, HCWs seem to be incentivized by their professional ethics and support from their family and peers. While health system constraints already jeopardized HCWs' functionality and motivation, they were further debilitated by the impacts at the personal front that included their own and family's safety, living isolated from families, increased discrimination, and stigma at their community and at the household (rental properties). Also, HCWs expressed concerns about the changes in their clinical practice, particularly as the way they were applying 'don't touch'measures. Recommendations from HCWs echoed around the need for additional resources (PPE, trained health human resources, equipment, and isolation and quarantine places) in 
addition to additional incentives and security of HCWs. Below we describe each of the components of health system preparedness and its impacts on HCWs.

\section{A. Government's efforts in health service preparedness for COVID-19}

\section{Initiation of the Incidence Command Activating System}

Nepal's Ministry of Health and Population initiated the Incidence Command Activating System that drafted various guidelines for COVID-19 case management and disseminated COVID19-related information to the public through print, electronic, and telecommunication media. However, a policymaker at the federal government acknowledged that there were lapses during the initial phase of crisis management, mainly due to inexperience in handling such emergencies.

We do not have any past experience of handling this type of pandemic, so we were not organized in our approaches for preparedness during January and February. However, starting in March, we have tried our best to be more organized and more systematic using a one-door policy. The Incidence Command System, which is initiated during emergencies, has been activated in the Ministry of Health and Population. Incidence Command System Officers have been designated responsibilities. Health experts and various other field experts, including government and non-government organizations, form a cluster and work together.

(Policymaker, male, federal government)

\section{Public health measures - social distancing and lockdown}

The Government of Nepal imposed strict lockdown across the country, cancelled all domestic and international flights, and sealed the open border with India. Despite these measures, there were large influxes of migrant workers through illegal routes. In response, the provincial government took additional steps such as large-scale testing in border districts and deploying border security forces to prevent such influx.

We have been doing testing on a large scale in bordering districts...... There is suspicion that some people might have entered Nepal through illegal routes. But since yesterday armed police have been deployed along the border and they are present every 100 meters. So now it is not possible to enter Nepal through any illegal routes. Moreover, the elected political leaders would notify the government if any new people are seen in their respective areas. And we are working out on enforcing quarantine and testing those people for COVID-19 infection.

(Policymaker, male, Province 1) 
Although the policymakers frequently emphasized 'lockdown' as the government's major public health intervention, they less frequently acknowledged the role of social distancing measures.

\section{Dedicated COVID-19 healthcare facilities}

In response to the COVID-19 related national emergency, the government first mapped out all the public hospitals and then categorized them into three levels based on their capacity and infrastructures, and designated each to treat mild, moderate, or severe COVID-19 cases.

We have mapped out how many hospitals we have in the nation, their locations, their bed capacity. Secondly, we categorized hospitals in three different categories. We have chosen COVID clinic hospitals and further divided them into three levels: Level 1, Level 2, and Level 3. We have defined the responsibilities of each level of the hospital. While Level 1 hospitals are responsible for managing mild cases of positive COVID cases, Level 2 hospitals are responsible for severe cases where ICUs and ventilators are needed. Level 3 hospitals are responsible for managing the severe cases of COVID with other comorbidities.

(Policymaker, male, federal government)

The reason behind this categorization of hospitals were reported to prevent and control the overwhelming and unsystematic attendance at hospitals. Nonetheless, none of the policymakers spontaneously appreciated the potential transmission of diseases to other patients who may be simply attending the hospital for regular visits, or other diseases.

Few policymakers also shared the government's plan to expand the resources by partnering with private healthcare facilities.

We have setup COVID-19 dedicated hospitals for the increasing number of coronavirus cases. If this is not sufficient, we will use the infrastructure of medical colleges in our province for the management of coronavirus patients.

(Policymaker, male, Gandaki Province)

\section{Guidelines for the management of suspected COVID19 cases}

As a part of the preparedness and to ensure the uniformity in the management of COVID-19 cases, Nepal's health ministry drafted and disseminated the guidelines for clinical and public health management of COVID-19. These guidelines, for instance, provided details for the clinical management of the cases of COVID-19, including prevention, treatment, and control measures.

The government of Nepal has prepared a dozen guidelines for the COVID-19 management, which includes prevention, treatment and control measures, management of quarantine and isolation, and proper use of 
PPEs. We have shared these guidelines to all our health workers. We regularly conduct meetings using videoconferencing to disseminate information and updates on the situation related to the COVID-19, and best practices followed in the region.

(Policymaker, male, Gandaki Province)

Although, such preparedness was appreciated by the HCWs, but they accentuated the looming constraints and gaps within the health system that could hinder effective management of COVID-19 cases.

\section{B. Gaps in healthcare/services preparedness}

\section{Lack of integrated approaches and coordination between three tiers of the public health system and between public and private healthcare facilities}

HCWs highlighted several gaps. An important gap was the overarching mechanism of functioning in the new federal health system where the coordination between the three tiers of the governmental health system (i.e., federal, provincial and local) was 'poor,' and the local and provincial healthcare facilities lacked adequate resources to operate effectively and independently. These constraints were reported to be operating in the background and thus affected the management of the COVID-19 pandemic. In addition to these operational constraints, lack of trained human resources (lacking specialized capacities), particularly as COVID-19 is a new disease, also added to the poor management.

There are three tiers of government performing their responsibilities; however, in a situation like now, there is no integrated approach taken. They have different strategies, and I do not think the coordination is quite good as just one level being good is not enough in this situation. Management is weak; there is a Corona Control Committee of ministers for integrated works that is not working out. We should make a team of doctors, public health professionals, motivating them through an integrated approach. The government staffs are not sufficient; we need participation from the private sector as well. We should move forward, making one team, which is lacking in the current state.

(Doctor, male, tertiary hospital, Kathmandu)

Lack of coordination between the three tiers of government was also strongly echoed by the policymakers at the provincial level who challenged the current federal system comparing it with the previous integrated system where coordination was more efficient. Against the backdrop of tenet underpinning the devolvement of responsibilities to provincial and local government for the management of current pandemic, policymakers seem to revive the previous coordination mechanism through personal contacts and communication with the federal government. 
It was difficult because, in this federal setup, there is a lack of system anchoring these three tiers of government in health. We relied on our personal relations to navigate the issues of governance. The health of the population should be the responsibility of the central government in this type of epidemic. The health system before the federal setup would have worked more efficiently in this type of epidemic.

(Policymaker, Male, Province 1 Government)

In addition to the inefficient coordination between the three tiers of government, lack of engagement and partnership with private hospitals seemed to be overlooked aspects of the current management of COVID19 pandemic. The current approach of the government in preparing public healthcare facilities alone also seemed to de-functionalize private healthcare facilities' potential capacity and contribution to COVID-19 management. Among several ramifications of obviating the partnerships with private hospitals, high referrals to public health facilities were one of the prominent concerns. In addition, lack of partnerships and delegation to private hospitals have rendered them a paradoxical complacency to defer patients and stay away from the responsibilities to the extent that patients who could be suffering from non-COVID related conditions were simply deferred instead of establishing the diagnosis and providing treatment.

Private hospitals are referring to excessively... all cases of fever.....you know, [fever] it could be a symptom of 100 other diseases, to our government hospital. Patients are having difficulty in finding immediate service for their ailments. Elderly people without respiratory symptoms, without fever are also finding difficulty in receiving treatment for suspicion of COVID. As these patients could be simply suffering from COPD [Chronic Obstructive Pulmonary Diseases], Asthma, or other chronic conditions.

(Staff nurse, female, public hospital, Gandaki Province)

\section{Inadequate number of frontline health workers}

HCWs endure a dire situation in Nepal, mostly in terms of their vulnerability in finding jobs and appropriate salary. For the current pandemic, the government of Nepal announced short-term (for three months) vacancies, but lack of a single applicant suggests it to be unpopular for most of the clinicians. Historically, Nepal's health system has faced a shortage of human resources; and permanent positions for doctors and nurses remain unfulfilled. Aversion among HCWs for government-sponsored jobs, especially during this health crisis, may aggravate the outbreak.

No, the numbers of doctors we have are all working, and if one of them gets infected, we don't have a substitute. The government also has not been able to add human resources. In the present context, the government has announced a vacancy for three months that almost implies 'use and throw.' So, if the government is serious about fighting the situation [current pandemic], ......should hire doctors permanently. My contract has a month remaining, and I do not think I will continue after. We have worked 
in a situation of crisis; the government should consider that and provide some benefits. There hasn't been a single applicant for three months vacancy.

(Medical officer, male, tertiary hospital, Kathmandu)

\section{Limited equipment/resources (space, equipment, and facilities)}

HCWs in Nepal expressed grave concerns towards the limited resources such as the numbers of isolation and treatment beds, laboratories for prompt diagnosis, instruments, ventilators, and PPEs. Importantly, the chronicity of deprivations of infrastructure and equipment was reported to be accentuated during the crisis. Lack of adequate capacity for lab testing and contact tracing were major concerns. Also, these capacities were more prominent in the peripheral regions of Nepal.

The unavailability of proper equipment and infrastructure is not new to Nepal. However, the lack of infrastructure, particularly space for isolation wards, beds become much more obvious during the time of crisis. The preparedness of health facilities is not up to the mark even at normal times. We do not have enough ventilators, even for normal times in our country. The ventilators that we have is not enough for patients with other conditions. We cannot deny ventilators for those patients and use them to treat COVID patients. And more importantly, there are no adequate PPEs for clinicians.....also, the question remains who should get PPE. What about sweepers and housekeepers? I am also not confident about the current laboratory capacity to meet the tests. The central lab already struggles to meet the test-demands, what about outside Kathmandu?

(Resident doctor, male, tertiary hospital, Kathmandu)

In terms of availability of resources, there were a few disconnects and dissonance between HCWs and policymakers. The policymakers conceded limited infrastructure to deal with the current crisis but assured that the government is trying its best to procure the necessary equipment.

Although our frontline health workers are well-prepared to deal with COVID-19 cases, there are several logistical challenges at the same time. I must admit that there are not enough PPEs, ventilators, N95 masks, cardiac monitors, and ICU beds in our province and their access, too. However, the government is trying its best to establish Government to Government (G2G) deals to import essential equipment from neighbouring countries.

(Policymaker, male, Gandaki Province)

\section{Motivation of frontline health workers}

\section{Professional ethics}


Amidst the context of deprived safeguards (such as PPE) for HCWs, their high motivation to work were perceived to be emanating from professional ethics. Also, HCWs felt that their responsibility was irreplaceable and felt a moral obligation to serve the patients.

We are nurses by profession. We have joined this profession to serve ill people. If we do not take care of sick, who else is there to take their care? So, our nursing ethics has motivated us to keep working.

(Staff nurse, female, provincial hospital, Province 2)

\section{Global appreciation of health workers, family, and peer support}

Frontline HCWs also mentioned how they were motivated to work referring to the global appreciation for their work during the pandemic. HCWs also compared their work during the pandemic akin to a frontline warrior during disasters. In addition, importantly, the respect and appreciation they garnered from their family and peers who knew their profession and appointment at hospitals also motivated them to work.

It is our duty as healthcare providers. Health workers have been called 'frontline warriors'....... during this pandemic. We are mainly motivated by the exemplary work of paramedics, nurses, and doctors in other countries, as seen on various social media. In fact, peers and family members know where we work, their constant encouragement, and respect for our work, and more so during this time also drives me to work.

(Resident doctor, male, tertiary hospital, Kathmandu)

Despite their high motivation, few HCWs were concerned about the disproportionate burden assigned at work, particularly referring to how seniors and consultants burdened the responsibilities (of patient care) to juniors.

\section{Impact of COVID-19 on frontline healthcare workers}

\section{Concerns about personal and family safety}

HCWs reported heightened fear of contracting COVID-19 while attending patients, particularly because of its high infectivity and burgeoning reports of infections and deaths among the frontline HCWs globally. In addition, the potential to transmit the disease to their family members further aggravated their anxiety.

It has impacted my mental health. We hear the news about how COVID-19 is spreading worldwide, and how health workers are contracting the infection, and there is the fear of transmitting to our families if we unknowingly contract the infection."

(Resident doctor, male, tertiary hospital, Kathmandu) 
Additionally, the unavailability of PPE was often referred to as the chief stressor in the workplace. Lack of PPE was a serious concern to an extent that they built their makeshift PPEs despite having doubts about its effectiveness. Contrary to the standard protocols for preventing cross-infection that mandate changing into a new set of PPEs before handling another patient/suspected case, many HCWs reported using the same PPE throughout the day while attending different patients due to the shortages.

We haven't seen how standard PPE looks like. We have PPE made by the hospital that includes plastic gowns, surgical mask, surgical cap, there are no N-95 masks and shoe covers. We are using locally made ones just for the sake of our satisfaction.....and we don't know if it can offer us protection.

(Resident doctor, female, tertiary hospital, Kathmandu)

\section{Isolation from families}

Frontline HCWs feared that they could inadvertently infect their family members. Few HCWs, particularly breastfeeding mothers and those with small kids, were despaired of how they had to restrain themselves from approaching their family members when returning from work.

I have a small child. After returning from [hospital] duty, I cannot go straight away to my child.......

(Staff nurse, female, tertiary hospital, Kathmandu)

Frontline HCWs working at COVID-19 designated health facilities were mandated to live in temporary hostels and were allowed to return home only after 14 days of self-isolation, following the last exposure with a COVID-19 patient. If and when these HCWs saw suspected patients more frequently, their quarantine could perpetuate abstaining from visiting their family members.

Our Province 1 government has set up a quarantined hostel for those frontline health workers working in these dedicated hospitals. The health workers in these COVID-19 dedicated hospitals can leave the premises of these hostels only after 14 days' quarantine and after having a negative COVID-19 test.

(Policymaker, male, Province 1)

\section{Discrimination and stigma}

HCWs, living in rented dwellings, faced discrimination from their landlords. They were verbally abused, labelled as 'disease carriers,' and even asked to vacate the rental unit. Fear of bringing virus from the hospital, infecting the household members, and contaminating the whole property were some of the reasons for such discriminatory acts. While these acts have been of great concern, the Ministry of Health and Population issued counter orders to stop such discriminate. 
My friend lives in a rented room near the hospital. One day after finishing her duty, when she returned to her room, the house owner started washing the gate and the door carpet with soap and water and sprayed some religious powder on the entrance.

(Resident doctor, female, tertiary hospital, Kathmandu)

I live in a rented room, but recently I was asked not to come to my room after working in the hospital all day.

(Medical officer, male, tertiary hospital, Kathmandu)

\section{Change in patient-provider relationships}

HCWs have adopted new methods of clinical interactions where their traditional way of examining patients has been challenged due to COVID-19. For instance, the 'don't touchpractice' entailed minimizing the physical interactions with patients, which essentially meant that HCWs would have to compromise the proper physical examination of the patients. Such a practice not just discouraged HCWs from seeing patients in person, particularly when they are febrile, it also meant that the patients, with fever and respiratory symptoms, had to visit multiple hospitals for healthcare.

We are avoiding examination and providing only symptomatic treatment to patients [with fever] and this can affect patients and physicians as well....they [patients] may have to visit other hospitals.

(Doctor, male, tertiary hospital, Kathmandu)

\section{E. Recommendations}

The majority of the HCWs and policymakers stressed that the government needs to ensure adequate stock of PPE and other infrastructure such as isolation beds. They also emphasized that HCWs must be incentivized through various means.

Ensuring adequate stock and supply of PPE and other medical equipment, appropriate management of isolation wards, training for frontline health workers, incentives and health insurance of health workers to keep them motivated are some of my recommendations.

(Medical officer, tertiary hospital, Kathmandu)

All participants resonated that the government needs to learn from the current crisis and strengthen the health system to fight effectively against such crises in the future. A health worker also cued towards a chronic problem of failing to learn from the past failures and high acceptance of such a tendency. 
We have learnt a huge lesson from this pandemic. The basic step of prevention, which we did not follow properly, has become a routine by now. In countries like China and Taiwan, who dealt with SARS, had an excellent outbreak response system and were prepared for emergency situations like these. Due to which, the transmission was limited. Similarly, we should take this opportunity and learn from this regarding how to handle the cases.

(Resident doctor, male, tertiary hospital, Kathmandu)

\section{Discussion}

\section{Summary of findings and current preparedness}

This study illustrates the characteristics, drivers and impacts of Nepal's preparedness towards COVID-19. Although Nepal had adequate time and opportunities to act sooner, the poor anticipation and inadequate preparedness delayed the building of necessary infrastructure, responsibility distribution and resource allocation. While policymakers expressed a few preparedness measures such as establishing COVID-19 specific hospitals, and implementing complete lockdowns, these were conceded to be futile by both policymakers and frontline HCWs as these preparedness gradually faltered, mostly due to poor systemic coordination between three tiers of governments and constraints in HCWs, logistics, infrastructure, and laboratory facilities to maintain testing coverage. Despite the myriad of challenges at the professional front with inadequate PPE and at the personal front with acts of discrimination, and working in isolation from families, HCWs seem to be intrinsically motivated to serve at the frontline. The findings from this study bear urgent implications for health system preparedness towards the COVID-19 and for future disasters.

\section{Gaps in healthcare/services preparedness}

One of the overarching problems that seemed ubiquitous in Nepal's preparedness was the gaps in coordination between federal, provincial and local level health system under the federal governance structure [4], that inevitably affected the preparedness and response towards COVID-19. Lack of coordination between these three-tier governance meant a lack of clarity on responsibility division, poor logistic supply chain management and deprivation of adequate (trained) health human resources at the provincial and local level. While public hospital's preparedness before COVID-19 pandemic was already

suffering from the lack of resources [4], the government's failure to engage with the private health sector as well as the non-health sector during this crisis seemed even more detrimental. Private health infrastructure in Nepal constitutes a large landscape in Nepal's health system, thus engaging and utilizing their space, and resources could significantly palliate the burden of public healthcare infrastructure [16]. Additionally, non-health infrastructure can also be repurposed for COVID-19. Specifically, sharing and devolving testing and tracing, space for quarantine, isolation beds and intensive care could have been 
negotiated for the current crisis. For example, Ecuador's Government collaborated with SENSEable City Laboratory and repurposed shipping containers into temporary makeshift intensive care units [17].

Health workforce shortages have been a chronic problem in Nepal, and yet it has been constantly overlooked over the years [5]. Nepal has 0.17 doctors and 0.5 nurses per 1,000/population. This cumulates to 0.67 doctors and nurses per 1,000 /population, and is well below the WHO's minimum benchmark of 2.3 doctors, nurses and midwives per 1,000/population [18]. This study clearly highlighted how the background deficit in HCWs suddenly appeared as a major foreground hindering the preparedness of the current pandemic. This was further evident from the fact that the short-term vacancies for doctors went unfulfilled and were considered to be exploitative ('use and throw'). Such measures are likely to compound current and future retention of HCWs and their interest in truly serving in public hospitals.

Frontline HCWs in this study who expressed high motivation to serve during such a crisis, were however, anxious because of the lack of adequate safety gears, particularly the PPE. HCWs working in the frontline were vulnerable to an extent that they used improvised surrogate-PPE out of rain coats, scarfs, and visors made from transparent sheaths [19]. While the government of Nepal is quite cognizant about the shortage of health human resources, failure to appreciate it at the budgetary announcement for the current fiscal year 2020/21 has been despairing. The budget has continued to ignore the need for health human resources, professional supervision and better opportunities for specialized training. However, allocating budget for establishing 50 bedded hospital in each province and 300 bedded at the federal level for management of infectious disease outbreaks are appreciable [20].

\section{Motivation of the frontline health workers and impact of COVID-19 on frontline healthcare workers}

HCWs in Nepal have shown a great deal of resilience despite more than a third had anxiety and depression during the current COVID pandemic [21]. HCWs identified professional ethics, global appreciation of HCWs' contribution to the pandemic and importantly, family members and peers' support and encouragement as major motivating factors for serving patients, even without proper infrastructure and PPE. A previous study from Nepal have also highlighted how the individual determination, a sense of responsibility to the community and professional duty among HCWs were motivating factors to serve during the crisis [22]. Also, HCWs' sacrifices in terms of how they maintain social distance with their family members, particularly in Kathmandu, where they cram in a rented room/apartment is difficult to imagine and can have psychological and social impact [23]. Nonetheless, unfortunately, HCWs in Nepal suffer from a multitude of problems at various levels. While the acts of discrimination towards HCWs from their landlords are quite specific to the current pandemic and has been associated with the fear of infection, and transmission; the sporadic incidents of verbal and physical abuse at the healthcare institutions even during this pandemic has been horrendous [24]. Such discriminatory practices can lead to low self-esteem, stress and anxiety among HCWs - as shown by studies elsewhere [25]. 
One notable outcome that HCWs seem to express was the new normal in their clinical practice as the way they had to adjust to 'don't touch' practice. Although physical examination and thorough investigation are part of the clinical practice' integrity, lack of PPE, safety concerns and subsequent fear of contracting the COVID-19 look discerning. Nonetheless, the adversity of such a practice can go in either direction. For instance, patients may perceive increased social distance with the HCWs, thus may attribute such acts as mal-practice or may simply perceive such practice as a precautionary measure to prevent disease transmission. Crushed between these extremes, HCWs, unfortunately can face the predicament of whether to conduct physical examination (without PPE) or face the allegations of malpractice, which could potentially invite patients' potential resentment, disappointments, and violence.

\section{Strengths and limitations}

This is the first study to explore the characteristics of Nepal's health system preparedness, factors affecting it and its impact on frontline HCWs. As such, this study also represent as the first report from low income countries. This study utilized remote interview methods using telephone or internet based tools which may have missed nuances and subtle expressions characteristics of the face to face interview. Nonetheless, this study covers a wide range of respondents from all across the country, thus has a diverse range of opinions. The sample composition was uneven, with disproportional participation of HCWs from tertiary level hospitals in Bagmati province that may have skewed our findings. Other frontline workers, such as dentist public health professionals, laboratory persons, ambulance drivers, and housekeeping staff at the hospital, could not be interviewed and was beyond the scope of this study.

\section{Conclusion}

One of the prominent lessons learned from the COVID-19 pandemic that could be relevant for Nepal and elsewhere is to have pre-emptive and adequate preparedness early on. Although a few preparedness measures were implemented in Nepal, such as the designation of COVID-19 specific hospitals and lockdown, the maintenance and sustainability of these measures were not forethought. The identified gaps in Nepal's health service preparedness against COVID-19 identified in this study emanated from a. systemic lack of coordination between the governance system and $b$. a severe shortage of resources (human and non-human), to tackle the pandemic at all levels. Specifically, the lack of human resources, inadequate logistic chain management and laboratory facilities for testing COVID-19 appeared to have jeopardized the health system preparedness and escalated the pandemic in Nepal. Despite health and safety concerns, HCWs have shown a great deal of resilience and motivation to serve to their capacities.

\section{Abbreviations}

COVID-19: Corona Virus Disease 2019; HCWs: Health Care Workers; MMIHS: Manmohan Memorial Institute of Health Sciences; PPE: Personal Protective Equipment; IRB: Institutional Review Board; SSIs: Semi-Structured Interviews; WHO: World Health Organization 


\section{Declarations}

\section{Acknowledgments}

We are deeply grateful to all the respondents of this study who have been relentlessly contributing their efforts to curb the pandemic at various capacities.

\section{Funding}

There are no funding related to this work.

Availability of data and material

Data cannot be shared publicly because of the nature of qualitative data; it contains personal quotes and clues that may potentially lead to participants' identification. However, in compliance with the data access policy outlined by the Institutional Review Committee of Manmohan Memorial Institute of Health Sciences (https://www.mmihs.edu.np/irc.php), data is available upon reasonable request to the chair of the research department (e-mail: drdharmakhanal@gmail.com).

\section{Authors' Contributions}

NS, SRM, and BA conceived the study. NS, SRM, BA, BG, and SG designed the interview guide. NS, BA, SBM, BG, SB, NilS conducted the interviews. NS, BA, BG, RY, SB, and NilS transcribed the data. NS and BA analyzed the data. BA and NS prepared the first draft and was revised by SRM, BG, and SG. All other authors contributed significantly during the revision. All authors read and approved the final version of the manuscript.

\section{Ethics approval and consent to participate}

Institutional Review Board (IRB) at Manmohan Memorial Institute of Health Sciences (MMIHS), Kathmandu, Nepal (Registration number: 76/133) approved the study. All participants were briefed about the study objectives and procedures, and of potential benefits and harms in participation. Participants were informed of voluntary participation and their rights to withdraw from the interview at any point or to refuse to answer any questions. Because of the COVID-19 pandemic, verbal consent was deemed appropriate for the interviews by the IRB of MMIHS. Participants who provided verbal consent and permission to audio-record the interview were included in the study. Data were de-identified, and any identifying contents were replaced with specific codes throughout the transcription process to ensure participants' anonymity and confidentiality.

\section{Consent for publication}

Not applicable.

\section{Competing interests}


The authors declare that they have no competing interests. Bipin Adhikari serves as an associate editor at BMC Public Health.

\section{References}

1. Zhu N, Zhang D, Wang W, Li X, Yang B, Song J, Zhao X, Huang B, Shi W, Lu R et al: A Novel Coronavirus from Patients with Pneumonia in China, 2019. N Engl J Med 2020.

2. Worldometer: COVID-19 CORONAVIRUS PANDEMIC. Available online at: https://www.worldometers.info/coronavirus/\#countries (Accessed on 4 July, 2020). 2020.

3. Bastola A, Sah R, Rodriguez-Morales AJ, Lal BK, Jha R, Ojha HC, Shrestha B, Chu DKW, Poon LLM, Costello A et al: The first 2019 novel coronavirus case in Nepal. Lancet Infect Dis 2020, 20(3):279280.

4. Ghimire $1 \otimes U$, Shrestha $2 \rrbracket N$, Adhikari B, Meheta S, Pokharel Y, Mishra SR: Health system's readiness to provide cardiovascular, diabetes and chronic respiratory disease related services in Nepal: analysis using 2015 health facility survey.

5. Adhikari B, Mishra SR: Urgent need for reform in Nepal's medical education. Lancet 2016, 388(10061):2739-2740.

6. Mishra SR, Khanal P, Karki DK, Kallestrup P, Enemark U: National health insurance policy in Nepal: challenges for implementation. Glob Health Action 2015, 8:28763.

7. Pokharel S, Raut S, Rijal KR, Adhikari B: COVID-19 pandemic, public health preparedness in Nepal and one health approach. Disaster Med Public Health Prep 2020:1-3.

8. Marahatta SB, Paudel S, Aryal N: COVID-19 Pandemic: What can Nepal do to Curb the Potential Public Health Disaster? Journal of Karnali Academy of Health Sciences 2020, 3(1):1-14.

9. Situation Report_COVID-19 \#109 (as of 28 May 2020) [https://heoc.mohp.gov.np/update-on-novelcorona-virus-covid-19/]

10. Ministry of Health and Population: COVID-19 Update. Available online at: https://covid19.mohp.gov.np/\#/ (Accessed on 13th June 2020). 2020.

11. Nepali Times: Protecting those who protect us from the epidemic. Available online at: https://www.nepalitimes.com/here-now/protecting-those-who-protect-us-from-theepidemic/ (Accessed on 28th March 2020). 2020.

12. Lai J, Ma S, Wang Y, Cai Z, Hu J, Wei N, Wu J, Du H, Chen T, Li R et al: Factors Associated With Mental Health Outcomes Among Health Care Workers Exposed to Coronavirus Disease 2019. JAMA Netw Open 2020, 3(3):e203976.

13. Tong A, Sainsbury P, Craig J: Consolidated criteria for reporting qualitative research (COREQ): a 32item checklist for interviews and focus groups. International journal for quality in health care 2007, 19(6):349-357.

14. Sargeant J: Qualitative Research Part II: Participants, Analysis, and Quality Assurance. J Grad Med Educ 2012, 4(1):1-3. 
15. Saunders B, Sim J, Kingstone T, Baker S, Waterfield J, Bartlam B, Burroughs H, Jinks C: Saturation in qualitative research: exploring its conceptualization and operationalization. Qual Quant 2018, 52(4):1893-1907.

16. Acharya S, Ghimire S, Jeffers EM, Shrestha N: Health Care Utilization and Health Care Expenditure of Nepali Older Adults. Frontiers in public health 2019, 7:24.

17. The World Bank: Global Responses to COVID-19 in slums and cities. Available online at: http://pubdocs.worldbank.org/en/359311591302725959/June1-Response-to-COVID-in-Slums-andcities.pdf (Accesed on 15th June 2020). 2020.

18. Ministry of Health and Population: Human Resources for Health Nepal Country Profile. Available online at: https://bit.ly/2YxdZl3 (Accessed on 13th June 2020). 2013.

19. Shrestha GS: COVID-19 Pandemic: Shortage of Personal Protective Equipment, Use of Improvised Surrogates, and the Safety of Health Care Workers. Journal of Nepal Health Research Council 2020, 18(1).

20. The Kathmandu Post: Nepal's budget: Not quite all there. Available at: https://kathmandupost.com/editorial/2020/05/28/nepal-s-budget-not-quite-all-there (Accessed on 13th June, 2020). 2020.

21. Khanal P, Devkota N, Dahal M, Paudel K, Joshi D: Mental health status among health workers in Nepal during COVID-19 pandemic. Policy brief. Available online at: https://bit.ly/3dYiSdr (Accessed on 13th June 2020). 2020.

22. Raven J, Baral S, Wurie H, Witter S, Samai M, Paudel P, Subedi HN, Martineau T, Elsey H, Theobald S: What adaptation to research is needed following crises: a comparative, qualitative study of the health workforce in Sierra Leone and Nepal. Health research policy and systems / BioMed Central 2018, 16(1):6.

23. The Kathmandu Post: Health workers deployed at Covid-19 frontline deprived of their allowances. Available online at: https://kathmandupost.com/national/2020/06/07/health-workers-deployed-atcovid-19-frontline-deprived-of-their-allowances (Accessed on 13th June 2020). 2020.

24. My Republica: Doctors 'manhandled' at Nepal Medical College. Available online at: https://bit.ly/2XZpnXO (Accessed on 13th June 2020). 2020.

25. Lanctôt N, Guay S: The aftermath of workplace violence among healthcare workers: A systematic literature review of the consequences. Aggression and violent behavior 2014, 19(5):492-501.

\section{Figures}




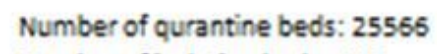

Number of isolation beds: 168

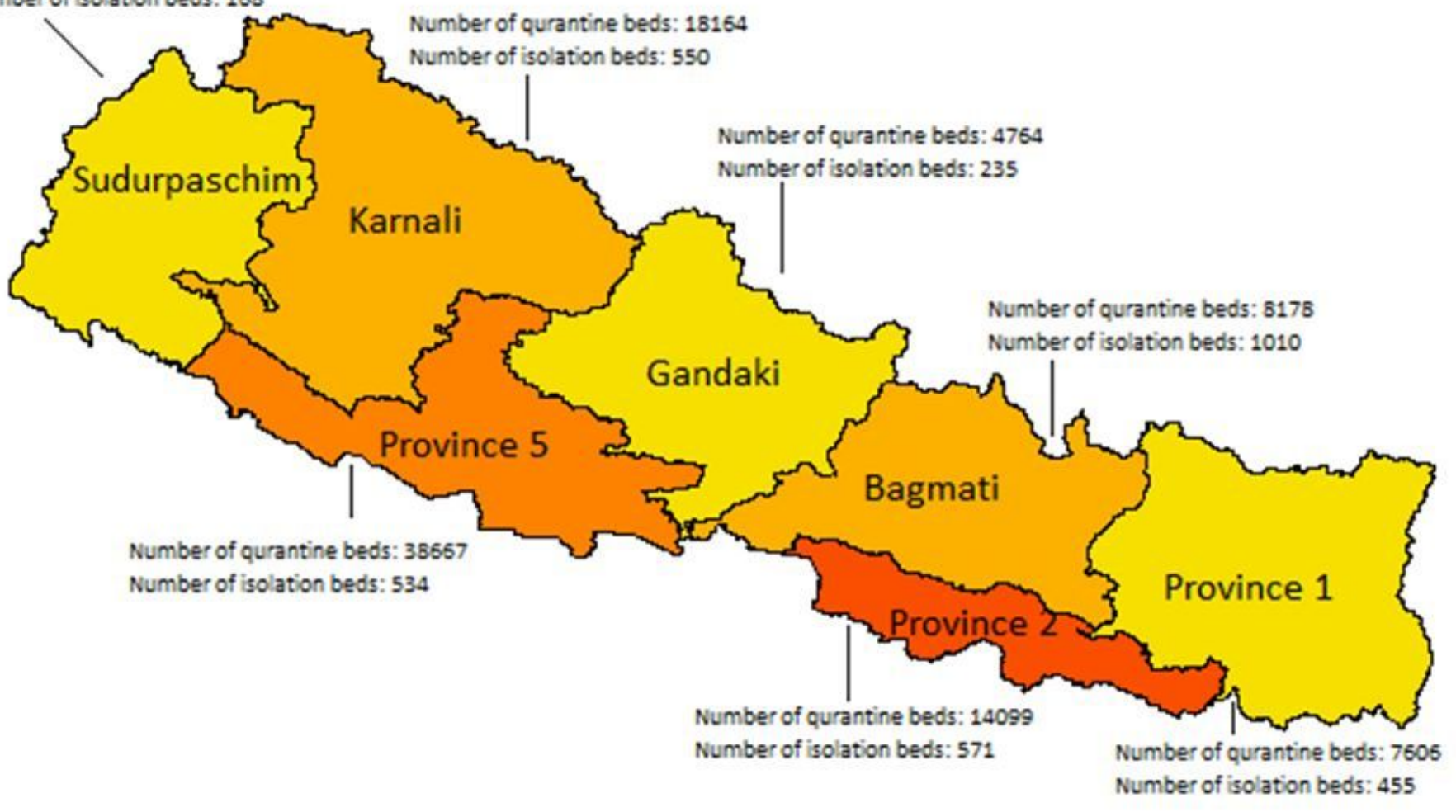

No. of COVID19 cases $\square<500$

$500-1000$

$1500-2000$

$>2000$

\section{Figure 1}

COVID-19 confirmed cases vs. health service preparedness by provinces of Nepal. This visualization includes 6,211 confirmed cases of COVID-19 reported until June 15, 2020. Geospatial map was created using GMAP procedure in SAS 9.4. The shape files were obtained from the Government of Nepal, Ministry of Federal Affairs and Local Development and were publicly available for unrestricted use (https://data.humdata.org/dataset/admin-shapefiles-of-nepal-mofald). 
- Incidence command activating system

Government's efforts in health service preparedness

- Public health measures

- Dedicated COVID-19 health care facilities

- Guidelines

Characteristics of health system preparedness, drivers and its impact on health care workers
Gaps in health care services

- Lack of integrated approaches and lack of coordination between stakeholders

- Inadequate health care workers

- Limited equipment/resources

\section{Motivation of frontline health workers \\ - Professional ethics \\ - Family and peer support}

Impact of COVID-19 on frontline health workers
- Concern about personal and family's safety

- Isolation from families

- Discrimination and stigma

- Change in patient-provider relationships

\section{Figure 2}

Health system preparedness to tackle the COVID-19 in Nepal: characteristics, drivers, and its impacts on healthcare workers.

\section{Supplementary Files}

This is a list of supplementary files associated with this preprint. Click to download.

- Appendix2SemiStructuredInterviewsGuide.pdf

- Appendix1COREQChecklist.pdf 